\title{
Efeito da Niclosamida no Controle de Girinos de Anuros na Propagação de Pós-Larvas de Carpa Comum (Cyprinus carpio Linnaeus, 1758 Var. Specularis)
}

\author{
Alvaro Graeff', Evaldo Nazareno Pruner², Marcia Mondardo Spengler ${ }^{3}$
}

\begin{abstract}
RESUMO - O objetivo desta pesquisa foi testar a influência de um molusquicida (niclosamida) no controle dos girinos, sem comprometer o desenvolvimento e a sobrevivência da carpa comum na fase de desenvolvimento do ovo até a idade de 28 dias. Foram conduzidos seis experimentos, envolvendo seis fases de desenvolvimento dos girinos e peixes (ovo, 7, 12, 21, 27 e 34 dias). O delineamento experimental foi inteiramente casualizado com cinco tratamentos $(0,00 ; 0,10 ; 0,15 ; 0,20$; e 0,25 ppm de niclosamida) e quatro repetições. Foram utilizados 20 aquários de seis litros, sendo todos abastecidos até o início do experimento, quando então era interrompido o fluxo de água. A eficiência da niclosamida no período de fertilização e eclosão de ovos de girinos e carpa não ocorreu. Na primeira semana de vida, os níveis de 0,25 ; 0,20; e 0,10 ppm mostraram efeito sobre os girinos aos 48, 65 e 115 minutos após a aplicação do produto respectivamente, mas apresentaramse sem efeito sobre as pós-larvas de carpa comum. A dosagem de $0,15 \mathrm{ppm}$ de niclosamida, aos 27 dias, tendeu a provocar maior mortalidade de girinos, com maior sobrevivência de pós-larvas de carpa comum, e a de 0,10 ppm, o melhor resultado na idade de 21 dias das pós-larvas de carpa comum e girinos.
\end{abstract}

Palavras-chave: alevinos, anuros, Cyprinus carpio, girinos, niclosamida

\section{Effect of the Niclosamida on the Control of Tadpole of Anurous in the Propagation of Post-Larvae of Common Carp (Cyprinus Carpio Linnaeus, 1758 Var. Specularis)}

\begin{abstract}
The objective of the research was to test the effect of a molusquicida (Niclosamida) in the tadpoles control and not to compromise the development and survival of common carps in the egg development until 28 days age. Six experiments were carried out and involved six tadpoles and fishes development phases (egg, 7, 12, 21, 27 and 34 days). A completely randomized experimental design, with five treatments $(0.00,0.10,0.15,0.20$ and $0.25 \mathrm{ppm}$ niclosamida) and four replicates, was used. Post-larvae and tadpoles stayed together in a density of 100 units of each from a nursery production. Twenty aquariums of six liters were all supplied until the beginning of the experiment, when the water flow was interrupted. Niclosamida efficiency did not affect the fertilization phase of tadpoles and carps eggs. In the first week, the levels of $0.25,0.20,0.10 \mathrm{ppm}$ affected tadpoles growth at 48,65 and 115 minutes after product application and showed no siginificant effect on the post-larvae of common carp. The $0.15 \mathrm{ppm}$ level of Niclosamida at 27 days of age tended to provok higher tadpoles mortality and higher post-larvae of common carp survival. The 0.10 ppm level of Niclosamida also tended to show the best result at 21 days in post-larvae of common carp and tadpoles.
\end{abstract}

Key Words: anuros, Cyprinus carpio, fingerlings, niclosamida, tadpole

\section{Introdução}

Os anfíbios são animais de sexo separados e no período da reprodução retornam ao ambiente aquático: no acasalamento o macho abraça a fêmea e ambos eliminam, ao mesmo tempo, seus gametas na água, caracterizando-se como uma fecundação externa.

Como resultado da fecundação, formam-se vários ovos envoltos por uma camada de gelatina. Se ficarem fora da água, esses ovos morrem, pois não têm nenhum revestimento especial que evite a perda de água.
Do ovo surge uma larva, chamada girino, que é um organismo tipicamente aquático. Possui uma grande cauda empregada para a natação e respira por brânquias. $\mathrm{O}$ girino sofre metamorfose, dando origem ao adulto (LOPES, 1996). Os anfíbios são classificados em três grupos: àpodes, urodelos e anuros. Este estudo se limitará ao último grupo, os anuros, ao qual pertencem os sapos e as pererecas durante a fase de ovo até a perda da cauda, na qual se definem os membros locomotores e a formação dos pulmões, razão pela qual não são mais considerados animais aquáticos.

\footnotetext{
${ }^{1}$ Médico Veterinário CRMV SC-0704 - Nutrição/EPAGRI - Estação de Piscicultura de Caçador. E.mail: agraeff@epagri.rct-sc.br

${ }^{2}$ Médico Veterinário CRMV SC-0401 - Reprodução/EPAGRI - Estação de Piscicultura de Caçador. E.mail: pruner@unc-cdr.rct-sc.br

${ }^{3}$ Engenheiro-Agrônomo CREA 10-26092, M.Sc., Estatística/EPAGRI - Estação Experimental de Caçador. Fone: (0xx49) 563-0211, Caixa Postal 591 CEP 89500 - Caçador, SC. E.mail: mmondardo@epagri.rct-sc.br
} 
Em função de o ciclo biológico dos peixes ser concomitante com os anuros, pois o início da propagação dos mesmos se dá na primavera, quando a temperatura da água ultrapassa os $18^{\circ} \mathrm{C}$, em comportamento animal este ciclo se enquadra nos ritmos dependentes da temperatura (CARTHY, 1980). O fato de a qualidade da água e alimentação de alto valor biológico atraírem os anuros estimula a desova por parte dos sapos e pererecas, promovendo uma competição por alimentação na ordem de $30 \%$ ou mais (PRUNER, 1984). Também na área de manejo, diminui-se em até $18 \%$ o índice de sucesso na produção de alevinos e eleva-se a mãode-obra para selecionar peixes dos girinos, no momento da venda (PRUNER, 1984).

A procura por métodos, manejos ou produtos que permita controlar estas perdas, sem causar danos ao meio ambiente, induziu à utilização de diversos produtos biológicos ou não em experimentação (TAMAS e HORVÁTH, 1976; JUAREZ, 1983; OPUSZYNSKY ET AL, 1984; FIGUEIREDO et al, 1990. Também tem-se utilizado óleo diesel sobre a superfície do viveiro, na quantidade de 20-40 1/ha (ROTHBARD, 1982; JHINGRAN e PULLIN, 1985), sem muita eficiência, e não recomendável por ser poluente.

Niclosamida (N-(2'-cloro-4'nitrofenil) - 5 clorosalicilanilida) é um princípio ativo, especialmente desenvolvido para controle dos caracóis (gasteropodos) de água doce que atuam como hóspedes intermediários dos organismos causantes da esquistossomose, fasciolose e outras afecções causados por trematodos. Também é indicado para uso humano para controle de teníase (Taenia solium e saginata) e da himenolepíase (Hymenolepis nana e diminuta) com inibição da fosforilação oxidativa dos mitocôndrios dos cestódios.

O objetivo deste trabalho foi testar a influência de um molusquicida no controle dos girinos, sem comprometer o desenvolvimento e sobrevivência da carpa comum.

\section{Material e Métodos}

Foram realizados seis experimentos, cada um para uma fase de desenvolvimento dos girinos e peixes (ovo, 7, 12, 21, 27 e 34 dias de vida). Para cada experimento foram utilizados 20 aquários de seis litros, sendo todos abastecidos até o momento do início dos tratamentos, quando então foi interrompido o fluxo de água, sendo povoados com pós-larvas de carpas comum (Cyprinus carpio L.) e girinos de sapo (Bufo sp.) na quantidade de 100 unidades de cada um em cada repetição.

O delineamento experimental foi inteiramente casualizado com cinco tratamentos $(0,00 ; 0,10 ; 0,15$; 0,20 ; e 0,25 ppm de niclosamida) e quatro repetições, com observações aos 20, 30, 40, 50, 60, 70 minutos e até 24 horas após o inicio do experimento. Em cada observação retirou-se o número de indivíduos mortos, anotados em planilhas específicas. Foram repetidos estes tratamentos com pós-larvas de carpas e girinos da mesma população, no período de 10 de novembro a 30 de dezembro de 1999, nas fases de: ovo, 7, 12, 21, 27 e 34 dias de vida. No sistema de produção normal após os 34 dias, as pós-larvas tornam-se alevinos e são entregues; os girinos têm sua metamorfose completa, quando o princípio ativo não tem mais efeito. Para a primeira semana de vida dos girinos, em que foram testadas as cinco doses do produto, foram obtidas as equações de regressão para a porcentagem de mortalidade em função das avaliações (20-70 minutos), para cada dose. A avaliação às 24 horas foi feita somente para observação prática do efeito residual.

A dieta oferecida foi a mesma que as pós-larvas de carpas comum (Cyprinus carpio LINNAEUS, 1758) recebem no sistema produção na Estação de Piscicultura/EPAGRI em Caçador/SC, conforme a Tabela 1.

A alimentação foi feita com as rações conforme Tabela 1, em quatro vezes por dia, de acordo com OGINO (1980) e NRC (1993), sendo ajustadas semanalmente. 
Tabela 1 - Composição das rações utilizadas no sistema de produção de alevinos de carpa comum (Cyprinus carpio L.), na Estação de Piscicultura/EPAGRI

Table 1 - Composition of the rations used in the system of fingerlings production of common carp (Cyprinus carpio L.), in the Station of Pisciculture/EPAGRI

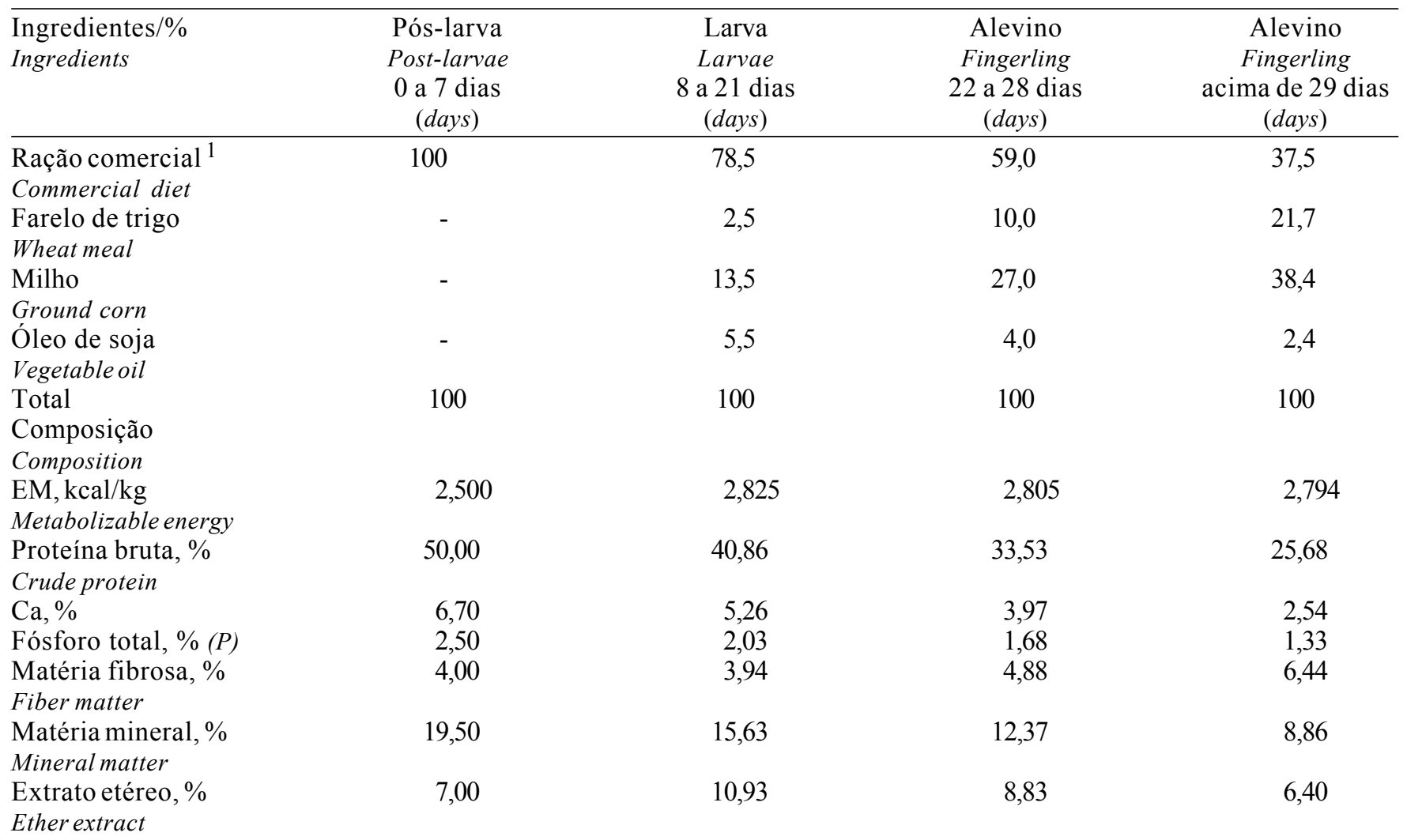

${ }^{1}$ Rações Nicoluzzi/Truta Alevinos (Ration Nicoluzzi/ Trout figerling): Vit. A, 20.000 UI; Vit. E, 150 mg; Vit. D, 4.000 UI; Vit. K, 5 mg; Vit. B 614 mg; Vit $\mathrm{B}_{12}, 60 \mathrm{mcg}$; Tiamina (Thiamin) $20 \mathrm{mg}$; Riboflavina (Riboflavin) $40 \mathrm{mg}$; Pantotenato de cálcio (Pantothenic acid) $100 \mathrm{mg}$; Colina (Choline) $1600 \mathrm{mg}$; Inositol (Inositol) 300 mg; Ac. fólico (Folic acid) 6 mg; Ac. nicotínico (nicotinic acid) 140 mg; Biotina (Biotin) 0,2 mg.

A temperatura da água, foi medida diariamente às 9 e 15 h, com um termômetro de coluna de mercúrio (esc: $-20 \mathrm{a}+70^{\circ} \mathrm{C}$ ). $\mathrm{O} \mathrm{pH}$, oxigênio dissolvido, gás carbônico, dureza, alcalinidade e amônia foram analisados semanalmente no Laboratório de Qualidade de Água/EPAGRI.

\section{Resultados e Discussão}

A temperatura da água durante o período experimental (Figura 1), no sistema de produção onde se retirava a amostra de girinos e pós-larvas de carpa comum, manteve-se entre 16,2 e $28,1^{\circ} \mathrm{C}$, ficando a média do período em $23,1 \pm 2,3^{\circ} \mathrm{C}$, próxima da relatada por ARRIGNON (1979), CASTAGNOLLI (1986), 24 e $28^{\circ} \mathrm{C}$ para carpas. A temperatura média no ambiente no período manteve-se entre 16,0 e $28,2^{\circ} \mathrm{C}$, ficando a média em $22,9 \pm 2,8^{\circ} \mathrm{C}$ dentro dos parâmetros normais para esta época do ano, em nossa região.

$\mathrm{Na}$ avaliação da qualidade da água, os parâmetros:
pH, oxigênio, amônia, gás carbônico, dureza e alcalinidade estavam dentro do preconizado por BOYD (1997) HUET (1978), ARRIGNON (1979), LUKOWICZ (1982), WOYNAROVICH (1985) e CASTAGNOLLI (1992).

A sobrevivência obtida dos alevinos, no fim do ciclo, nos dois viveiros de produção experimentais, de onde foram retiradas as amostras experimentais, foi de 5000 e 45.000 unidades ou seja 6 e $51 \%$, respectivamente das pós-larvas povoadas. Em contrapartida, a produção de girinos de várias idades, nos dois viveiros, foi de 40,0 e 5,0 quilogramas, respectivamente, demonstrando claramente a influência de concorrência por espaço e alimentação durante o cultivo.

Não ocorreu efeito da niclosamida nas doses testadas $(0,00 ; 0,10 ; 0,15 ; 0,20 ;$ e 0,25 ppm $)$ na fertilização e eclosão dos ovos de girinos (Bufos sp.) e peixes (Cyprinus carpio L.) e nem na sobrevivência na primeira semana de vida das pós-larvas de carpa comum. Para a mortalidade dos girinos na primeira semana de vida, ocorreu interação entre as doses de 


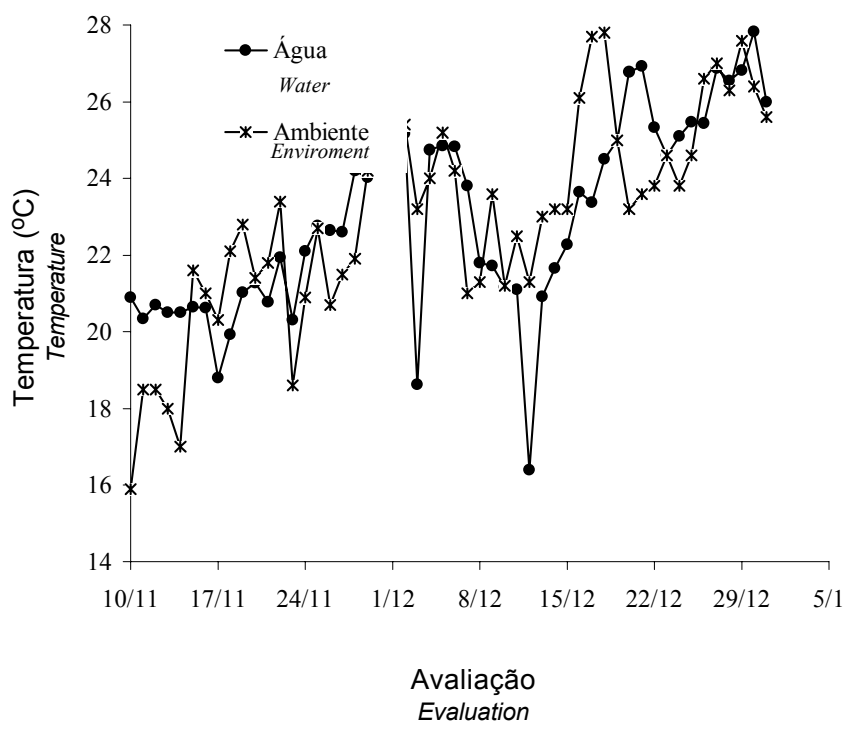

Figura 1 - Temperatura da água e do meio ambiente no sitema de produção.

Figure 1 - Temperature of the water and of the enviroment in the production system.

niclosamida testadas $(0,00 ; 0,10 ; 0,15 ; 0,20 ; \mathrm{e} 0,25 \mathrm{ppm})$ e os períodos de avaliação $(20,30,40,50,60$ e 70') e procedeu-se análise de regressão para a percentagem de mortalidade em função do tempo para cada dose (Figura 2).

A equação de regressão linear que melhor explica a eficiência da dose de 0,10 ppm é $Y=-24,6+1,08 X$; de acordo com esta equação, aos 70 dias em torno de $50 \%$ dos girinos estariam mortos. À dose $0,20 \mathrm{ppm}$ corresponde a equação de regressão quadrática $\mathrm{Y}=$ $-85,329+4,934 X-0,03214 X^{2}$; de acordo com esta equação a mortalidade é de $100 \%$ aos 65 minutos. À dose 0,25 ppm a equação de regressão quadrática é $\mathrm{Y}=-138,193+8,489 \mathrm{X}-0,0735 \mathrm{X}^{2}$ e, também de acordo com a equação, a mortalidade de $100 \%$ atingiria aos 48 minutos. Para a dose de 0,15 ppm não foi obtido bom ajuste dos valores observados nos modelos testados.

Analisando os resultados da Tabela 2, verifica-se tendência de diminuição da mortalidade com o aumento da idade dos girinos na dose $0,15 \mathrm{ppm}$, porém, após as 24 horas, os girinos com 27 dias estavam todos mortos. Na dose $0,10 \mathrm{ppm}$, constatou-se pequeno efeito nos girinos de 21 dias até os 70 minutos e, após as 24 horas, também ocorreu o efeito decrescente com as idades. $\mathrm{O}$ resultado explica-se pelo fato de, durante a metamorfose, os anuros necessitarem de
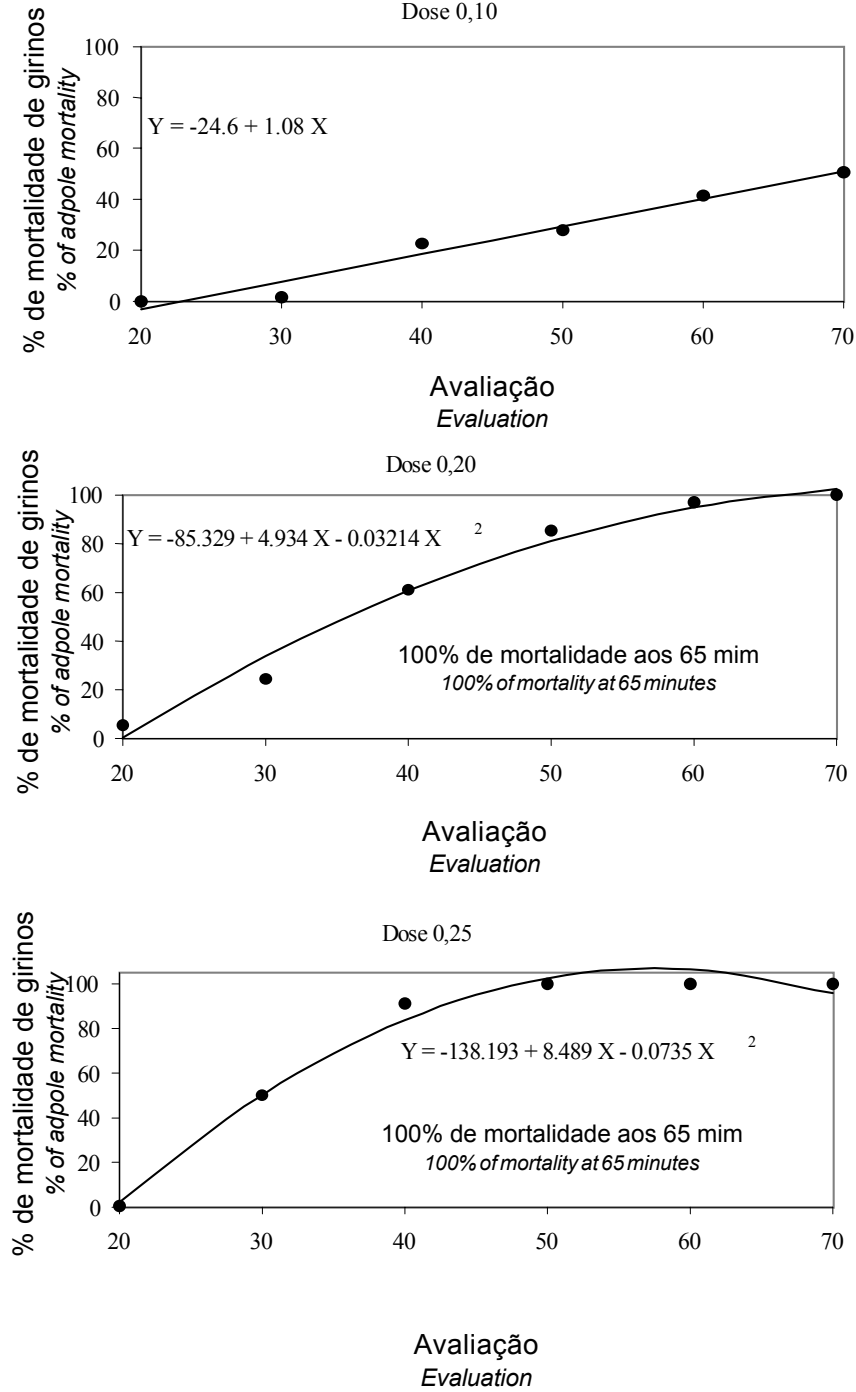

Figura 2 - Efeito da niclosamida na mortalidade de girinos com 7 dias de vida com doses niclosamida.

Figure 2 - Effect in mortality of tadpole in seven days with increase doses of niclosamida.

muito oxigênio difundido na água, pois nesta fase seu metabolismo é acelerado e, portanto, o consumo de oxigênio é elevado (VIZOTTO, 1978). A melhor eficiência ocorre aos 27 dias de vida do girino com a dose de $0,15 \mathrm{ppm}$ com $100 \%$ de mortalidade no primeiro dia de contato.

$\mathrm{Na}$ Tabela 3 observa-se sobrevivência baixa de pós larvas de carpa comum, somente na dose de 0,15 ppm na idade de 12 dias, após 24 horas de exposição ao produto.

Baseado nas observações das Tabelas 2 e 3, a melhor combinação de resultados entre a mortalidade de girinos e sobrevivência de carpas comum seria obtida na dose de $0,15 \mathrm{ppm}$ aos 27 dias de 
Tabela 2 - Efeito da niclosamida no percentual de mortalidade de girinos em função da idade e doses Table 2 - Effect of the niclosamida in the percentile of tadpole mortality in function of the age and doses

\begin{tabular}{|c|c|c|c|c|c|c|c|c|c|c|c|c|}
\hline \multirow{2}{*}{$\begin{array}{l}\text { Dose-ppm } \\
\text { Doses } \\
\text { Idade } \\
\text { Age }\end{array}$} & \multicolumn{4}{|c|}{0,15} & \multicolumn{4}{|c|}{0,10} & \multicolumn{4}{|c|}{0,00} \\
\hline & 12 & 21 & 27 & $34 *$ & 12 & 21 & 27 & 34 & 12 & 21 & 27 & 34 \\
\hline \multicolumn{13}{|c|}{ Avaliações em minutos } \\
\hline 20 & 0 & 0 & 0 & 0 & 0 & 0 & 0 & 0 & 0 & 0 & 0 & 0 \\
\hline 30 & 0 & 0 & 0 & 0 & 0 & 0,2 & 0 & 0 & 0 & 0 & 0 & 0 \\
\hline 40 & 12,5 & 0 & 0 & 0 & 0 & 0,2 & 0 & 0 & 0 & 0 & 0 & 0 \\
\hline 50 & 56,5 & 8,5 & 0 & 0 & 0 & 0,5 & 0 & 0 & 0 & 0 & 0 & 0 \\
\hline 60 & 78,0 & 46,5 & 4,5 & 0 & 0 & 0,6 & 0 & 0 & 0 & 0 & 0 & 0 \\
\hline 70 & 83,5 & 71,5 & 23,7 & 0 & 0 & 2,5 & 0 & 0 & 0 & 0 & 0 & 0 \\
\hline $\begin{array}{l}24 \mathrm{hs} \\
\text { hours }\end{array}$ & 99,5 & 87,0 & 100,0 & 14,0 & 78,6 & 62,0 & 32,0 & 3,5 & 0 & 0 & 0 & 0 \\
\hline
\end{tabular}

* Eficiência diminuída, imago fase de metamorfose com pouca dependência do meio aquático.

* Decreased efficiency, imago metamorphosis phase with little dependence of the aquatic way.

Tabela 3 - Efeito da niclosamida no percentual de sobrevivência das pós-larvas de carpa comum em função da idade e doses Table 3 - Effect of the niclosamida in the percentile of survival of the powders - larvas of common carp in function of the age and doses

\begin{tabular}{|c|c|c|c|c|c|c|c|c|c|c|c|c|}
\hline \multirow{2}{*}{$\begin{array}{l}\text { Dose-ppm } \\
\text { Doses } \\
\text { Idade } \\
\text { Age }\end{array}$} & \multicolumn{4}{|c|}{0,15} & \multicolumn{4}{|c|}{0,10} & \multicolumn{4}{|c|}{0,00} \\
\hline & 12 & 21 & 27 & $34^{*}$ & 12 & 21 & 27 & 34 & 12 & 21 & 27 & 34 \\
\hline \multicolumn{13}{|c|}{ Avaliações em minutos } \\
\hline 20 & 100 & 100 & 100 & 100 & 100 & 100 & 100 & 100 & 100 & 100 & 100 & 100 \\
\hline 30 & 100 & 100 & 100 & 100 & 100 & 100 & 100 & 100 & 100 & 100 & 100 & 100 \\
\hline 40 & 99,7 & 100 & 100 & 100 & 100 & 100 & 100 & 100 & 100 & 100 & 100 & 100 \\
\hline 50 & 99,7 & 100 & 100 & 100 & 100 & 100 & 100 & 100 & 100 & 100 & 100 & 100 \\
\hline 60 & 99,5 & 100 & 100 & 100 & 99,0 & 100 & 100 & 100 & 100 & 100 & 100 & 100 \\
\hline 70 & 99,5 & 100 & 100 & 100 & 99,0 & 100 & 100 & 100 & 100 & 100 & 100 & $100 \mathrm{~s}$ \\
\hline $\begin{array}{l}24 \mathrm{hs} \\
\text { hours }\end{array}$ & 20,5 & 82,0 & 100 & 99,5 & 87,5 & 99,0 & 100 & 99,0 & 100 & 100 & 100 & 100 \\
\hline
\end{tabular}

vida (100\% mortalidade dos girinos e $100 \%$ sobrevivência das pós-larvas), embora não tenha sido possível realizar esta comparação estatisticamente. A dose de $0,10 \mathrm{ppm}$ deverá de preferência ser aplicada a partir de 21 dias de idade das pós-larvas de carpa comum e os girinos com menos de 21 dias (Tabelas 2 e 3 ).

\section{Conclusões}

A niclosamida nas dosagens utilizadas não apresentou efeito sobre a fertilização e eclosão dos ovos de carpas comum (Cyprinus carpio L.) e girinos
(Bufos sp).

Na primeira semana de vida das carpas comum, as dosagens utilizadas não tiveram efeito deletério, enquanto estimou-se que $100 \%$ de mortalidade dos girinos ocorreu após os 70, 65 e 48 minutos, após o contato nas doses de 0,$10 ; 0,20$ e 0,25 ppm, respectivamente.

Existe tendência de a dose de 0,15 ppm aos 27 dias ter provocado maior mortalidade de girinos, com a maior sobrevivência de pós-larvas de carpa comum.

A dosagem de 0,10 ppm de niclosamida também apresentou tendência de melhor resultado aos 21 dias das pós-larvas de carpa comum (sobrevivência) e 
girinos (mortalidade), após 24 horas de contato.

\section{Agradecimento}

À Empresa Bayer S.A., na pessoa do Dr. João Paulo Gomes, pelo fornecimento do produto.

Ao técnico em Agropecuária Roberto Dal Pont, responsável pelo Laboratório de Qualidade de Água, pela dedicação durante as etapas de trabalho de campo.

\section{Referências Bibliográficas}

ARRIGNON, I. 1979. Ecologia y piscicultura de águas dulces. Madri: Mundi-Prensa. 365p.

BOYD, C.E. 1997. Manejo do solo e da qualidade da água em viveiro para aquicultura. Campinas: Associação Americana de Soja. 55p.

CARTHY, J.D. 1980. Comportamento animal. São Paulo: EPU: Ed. Universidade de São Paulo. 79p.

CASTAGNOLLI, N. 1986. Piscicultura nos trópicos. São Paulo: Manole, 152p.

CASTAGNOLLI, N. 1992. Piscicultura de água doce. Jaboticabal: Ed. Funep. 189p.

FIGUEIREDO, G.M., SENHORINI, J.A. 1990. Influência de biocidas no desempenho da carpa comum (Cyprinus carpio LINNAEUS, 1758) e sobre zooplâncton, durante o período de larvicultura. Bol. Téc. CEPTA, 3(único): 5-22.

HUET, M. 1978. Tratado de piscicultura. Madrid: MundiPrensa. 705 p.

JHINGRAN, V.G., PULLIN, R.S.V. 1985. A hatchery manual for the common chinese and indian major carp. Manila: Asian development Bank; International Center for living Aquatic Resources Management. 191p. (ICLARM Studies and reviews, n.11).

JUAREZ, L.M., ROUSE, D.B. 1983. Acut toxicity of trichlorfon to juvenile freshwater prawn. Progr. Fish-Cult., 45(4): 214-216.

LOPES, S., MACHADO, A. 1996. A vida. São Paulo: Atual. (Ciências; $6^{\text {a }}$ Série). 103p.

LUKOWICZ, M.V. 1982. Intensive carp Cyprinus carpio L. rearing in a farm pond in southern Germany and its effects on water quality. Aquaculture Engineers, 1(2):121-137.

NATIONAL RESEARCH COUNCIL - NRC. 1993. Nutrient requirements of fish. N.A.P. Washington, D.C. 114 p.

OGINO, C. 1980 Protein requirement of carp and rainbow trout. Bulletin Japanese. Society Sciences fisheries, 46:385-388.

OPUSZYNSKY, K., SHIREMAN, J.V., ALDRIDGE, F.J. 1984. Environmental manipulation to stimulate rotifers in fish ponds. Aquaculture, 42:343-348.

PRUNER, E.N. 1984. Dados não publicados. Caçador: Estação de Piscicultura EPAGRI.

ROTHBARD, S. 1984. Induced reproduction in cultivated cyprinids - the common carp and the group of chinese carps: II. The rearing of larval and the primary nursing of fry. Bamidgeh, 34(1):20-32.

TAMAS, I., HORVÁTH. 1976. Growth of cyprinids under optimal zooplankton conditions. Bamidgeh, 28:50-56.

VIZOTTO, L.D. Aspectos técnicos da ranicultura. In: ENCONTRO NACIONAL DE RANICULTURA, 1, 1978, Brasília. Sumula... Brasília, 1978. p.27-60

WOYNAROWICH, E. 1985. Manual de piscicultura. Brasília: MINTER/CODEVASF. 71p.

Recebido em: 10/10/00

Aceito em: 05/07/01 